\title{
Arsenic: a potentially useful poison for Hedgehog-driven cancers
}

\author{
G. Praveen Raju
}

Department of Pediatrics, Weill Cornell Medical College, New York, New York, USA.

\begin{abstract}
Dysregulated Hedgehog (Hh) signaling has been implicated in a growing number of human cancers. To date, most antagonists of this signaling pathway that have been developed target the $\mathrm{Hh}$ receptor Smoothened. However, these are predicted to have minimal effect when the pathway is activated as a result of dysregulation downstream of this receptor. In this issue of the JCI, Beauchamp and colleagues provide preclinical evidence that arsenic trioxide, a drug FDA approved for the treatment of acute promyelocytic leukemia, inhibits the growth of Ewing sarcoma and medulloblastoma cells by targeting GLI family zinc finger (GLI) proteins, which are Hh signaling pathway components downstream of Smoothened.
\end{abstract}

\section{Arsenic's long history in medicine}

When you mention arsenic, many people think of its poisonous effects. The truth, however, is that although arsenic is associated with several toxicities in humans, including peripheral neuropathy, cardiomyopathy, and renal failure, this small molecule has been used as a medical therapy for over 2,000 years. Its use as an antileukemia agent dates back to the late 19th century, and it was only replaced by modern chemotherapeutics in the 1950s (1). Since then, arsenic compounds, primarily arsenic trioxide (ATO), have had a resurgence as chemotherapeutic agents. In the early 1990s, several clinical trials showed that ATO was of benefit in the treatment of acute promyelocytic leukemia (APL), with complete responses observed in 70\%-90\% of newly diagnosed patients and $90 \%$ of patients with recurrent or drug-resistant APL $(2,3)$. It has been proposed that the mechanism of action by which ATO mediates its beneficial effects in patients with APL is through degradation of the fusion protein that drives disease, promyelocytic leukemia/retinoic acid receptor $\alpha$ (PML/RAR) (4). However, since ATO does show some modest benefit in multiple myeloma and myelodysplastic syndromes (1), other mechanisms of action are possible. In support of this, it has been shown in vitro that arsenic compounds can inhibit NF- $\mathrm{KB}$ and activate JNK activity to exert their effects either through stimu-

Conflict of interest: The author has declared that no conflict of interest exists.

Citation for this article: J Clin Invest. 2011; 121(1):14-16. doi:10.1172/JCI45692. lation of differentiation or induction of apoptosis $(5,6)$. However, a role for arsenic compounds in treating solid tumors has not been well established.

\section{Hedgehog signaling in cancer and Smoothened antagonists}

The Hedgehog (Hh) signaling pathway, originally identified for its critical role in multiple developmental processes in Drosophila (7), has been shown to be activated in several human cancers, with evidence for mutations in the genes encoding Hh signaling pathway components in some cases. Two cancers in which a role for dysregulated Hh signaling has been particularly well characterized are basal cell carcinoma and the pediatric brain tumor medulloblastoma (8). Based on preclinical mouse modeling that recapitulates the biology of human tumors through tissue-specific Hh activation, several $\mathrm{Hh}$ antagonists have been developed through in vitro screening of chemical libraries and proven to be efficacious in preclinical studies both in vitro and in vivo (9). To date, most of these $\mathrm{Hh}$ antagonists have targeted the Hh receptor Smoothened (Smo). These are, however, of little benefit in situations in which Hh signaling pathway dysregulation is mediated by effects on signaling components downstream of Smo, such as suppressor of fused homolog (Sufu) or GLI family zinc finger (GLI) proteins, or by increased expression of GLI1 through Hh-independent mechanisms $(10,11)$. Furthermore, recent clinical studies with a Smo antagonist, GDC-0449, in an adult medulloblastoma patient showed relatively rapid development of resistance to the drug, primarily as a result of mutations in SMO that prevented GDC-0449 binding to the encoded mutant protein (12). These data suggest that alternative approaches to blocking Hh signaling downstream of Smo are needed if this pathway is to be successfully targeted for the treatment of cancer.

\section{An additional mechanism for ATO action}

In this issue of the JCI, Beauchamp and colleagues report their preclinical studies using ATO in Ewing sarcoma cell lines and xenografts as well as in medulloblastoma cell lines and a transgenic mouse model of medulloblastoma (13). Although medulloblastoma and basal cell carcinoma are the best-characterized cancers with dysregulated Hh signaling, the investigators also focused their studies on Ewing sarcoma, as they previously established the Hh target gene GLI1 as an important transcriptional target of the oncogenic fusion protein Ewing sarcoma breakpoint region 1 /Friend leukemia virus integration 1 (EWS/FLI1), which drives disease in many Ewing sarcomas (14). Importantly, Beauchamp and colleagues were able to show that ATO not only has antigrowth properties in these tumor types, but that its mechanism of action is likely through direct binding and inhibition of GLI1 and/or GLI2 transcriptional activity and not through other mechanisms, such as DNA binding ability or subcellular localization (Figure 1 and ref. 13). Furthermore, they showed that primary cilia, which play an important role in Hh signal transduction, were not required for GLI1 inhibition by ATO, supporting a mechanism of action that could enable the use of this compound in settings in which Hh signaling is dysregulated downstream of Smo, or even in Hh-independent settings in which the GLI genes are overexpressed through genomic amplification. To assess in vivo activity of ATO in Ewing sarcoma xenografts and medulloblastoma cell lines, Beauchamp and colleagues nicely showed a 


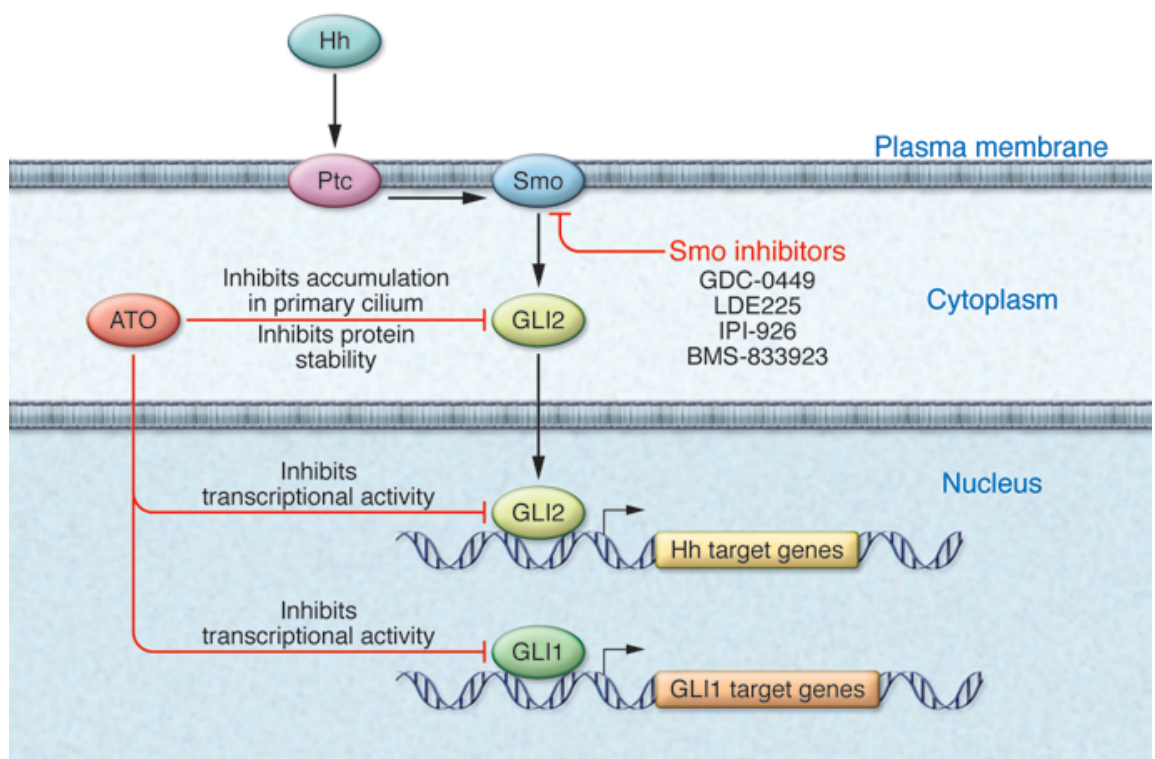

Figure 1

Proposed mechanisms of ATO action on Hh signaling pathway components. The schematic linearly shows activation of $\mathrm{Hh}$ signaling after binding of Hh ligand to the Patched (Ptc) receptor. This results in subsequent localization of the Smo transmembrane protein to the primary cilium, where further signal transduction occurs after proteolytic processing of GLI2 to an activator form, resulting in GLI2 nuclear translocation to transcriptionally activate $\mathrm{Hh}$ target genes. Smo inhibitors that are currently in clinical trials for human cancers are shown. ATO inhibits GLI1 and GLI2 proteins downstream of the Smo protein through similar, yet slightly distinct, mechanisms of action $(13,18)$.

correlation between the effects of ATO on growth inhibition and GLI protein levels: ATO affected growth in several tumor cell lines that have high levels of GLI1 or GLI2 protein expression, but little effect in cell lines with low GLI protein expression (13). Finally, using the ND2:SmoA1 transgenic mouse model of medulloblastoma, which expresses a constitutively activated Smo receptor within cerebellar granule cell precursors, they were able to show a marked survival benefit in ATO-treated animals. This result is particularly noteworthy, since a few studies in human patients treated with ATO after APL relapse within the CNS have suggested some benefit; a recent study of 45 patients who received single cycle ATO-based consolidation chemotherapy showed remission in 41 patients and only one patient who developed CNS relapse, with a median follow-up of 2.7 years (15). Conversely, others have shown that in two APL patients, when ATO levels were actually measured within the cerebrospinal fluid, ATO levels reached only $14 \%-18 \%$ of the serum ATO level, suggestive of relatively poor entry into the human brain $(16,17)$. With both the Ewing sarcoma xenografts and transgenic mouse model of medulloblastoma, no clear in vivo toxicity from ATO treatment was noted at the doses tested (13).

Recently, Beachy's group published a study demonstrating that ATO antagonizes Hh signaling primarily through interference with GLI2 (18). Although the main points of this study, that ATO interferes with GLI proteins in the context of a dysregulated $\mathrm{Hh}$ signaling pathway within cancer cells, are similar to those in the article by Beauchamp and colleagues (13), it is noteworthy that Beachy's group suggests that ATO functions by preventing GLI2 accumulation within the primary cilium with short-term treatment and by reducing GLI2 protein stability with long-term treatment (Figure 1 and ref. 18). These differences of whether the primary cilium is required for GLI inhibition or whether GLI1 and/or GLI2 is the primary ATO target might be related to the different cell types used or technical differences in the assays in the respective studies and will need to be sorted out in subsequent analyses.

\section{Future direction}

The study by Beauchamp and colleagues (13) and that from the Beachy laboratory (18), both of which implicate GLI proteins as molecular targets of ATO action, encourage further investigation of the role of $\mathrm{Hh}$ signaling in APL and other hematological malignancies in which ATO has been effective, including multiple myeloma and myelodysplastic syndromes. There has been little reported in the literature regarding the status of Hh or GLI activity within APL patients. One report of a small cohort of three patients with APL showed evidence of GLI1 expression in all three patients (19). Larger sample sizes will need to be investigated to confirm this, but the preliminary finding suggests that the beneficial effects of ATO in patients with APL might be the result of the drug targeting both PML/RAR and GLI proteins.

Although several other Hh signaling pathway antagonists, such as GLI-antagonist (GANT) small-molecule compounds, have been reported to function at the level of the GLI proteins, they will require significant preclinical testing to assess safety and efficacy before entering clinical trials, unlike ATO, an already FDA-approved treatment for APL (20). For non-CNS malignancies, such as Ewing sarcoma, that are Hh driven or express elevated levels of GLI protein, the ATO dosing regimen for APL can serve as a baseline for determining the appropriate dose, provided that GLI inhibition in preclinical studies demonstrates a benefit. However, with regard to using ATO for the treatment of medulloblastoma or other brain tumors, the pharmacokinetics of ATO dosing for adequate CNS penetration will need to be determined in order to minimize toxicity. As Ewing sarcoma and medulloblastoma are primarily found within the pediatric population, caution should be used when considering Hh signaling pathway blockade as a targeted therapy, particularly for younger patients. As the Hh pathway is crucial for both embryonic and postnatal development, Hh inhibition in younger patients may lead to teratogenic effects beyond typical drug toxicities. For example, in preclinical studies assessing Hh antagonists, even transient inhibition of Hh signaling in young mice resulted in profound and permanent defects in bone structure (21). Finally, when using xenografts to evaluate potential anticancer agents in preclinical studies, it is noteworthy that in many cases, the tumor still grows, albeit at a slower rate than that of control animals. Whether this is due to inadequate drug dosing or efficacy versus acquired resistance to the treatment over time is not clear; however, these find- 
ings should be taken into consideration and evaluated thoroughly in better preclinical in vivo tumor models, if available. As rapid treatment resistance was shown with use of a Hh antagonist in a medulloblastoma patient (12), further research into the mechanisms of ATO action on GLI protein function may provide insights into the use of this agent either at the time of tumor relapse after Smo antagonist treatment or in combination with Smo antagonists upfront. In conclusion, as resistance to the current therapeutic modalities that are being used to treat cancer is a major hurdle in the clinic, future preclinical studies similar to those described by Beauchamp et al. (13) may lead to additional treatment strategies for cancer patients who relapse despite aggressive interventions.

\section{Acknowledgments}

The author was a Fellow of the Pediatric Scientist Development Program (NICHD $\mathrm{K} 12$-HD00850) and is currently funded by a Clinical Scientist Award (NINDS K08NS066083).

Address correspondence to: G. Praveen Raju, Department of Pediatrics/Child Neurology, Weill Cornell Medical College, 525
East 68th Street, Box 91, New York, New York 10065, USA. Phone: 212.746.1279; Fax: 212.746.4001; E-mail: prr9001@med. cornell.edu.

1. Emadi A, Gore SD. Arsenic trioxide - An old drug rediscovered. Blood Reviews. 2010;24(4-5):191-199.

2. Sun HD, Ma L, Hu XC, Zhang TD. Ai-Lin I treated 32 cases of acute promyelocytic leukemia. Chin J Integrated Tradit West Med. 1992;12:170-172.

3. Soignet SL, et al. United States multicenter study of arsenic trioxide in relapsed acute promyelocytic leukemia. J Clin Oncol. 2001;19(18):3852-3860.

4. Chen GQ, et al. In vitro studies on cellular and molecular mechanisms of arsenic trioxide $\left(\mathrm{As}_{2} \mathrm{O}_{3}\right)$ in the treatment of acute promyelocytic leukemia. Blood. 1996;88(3):1052-1061.

5. Porter AC, Fanger GR, Vaillancourt RR. Signal transduction pathways regulated by arsenate and arsenite. Oncogene. 1999;18(54):7794-7802.

6. Kapahi $\mathrm{P}$, et al. Inhibition of NF-kappa B activation by arsenite through reaction with a critical cysteine in the activation loop of Ikappa B kinase. J Biol Chem. 2000;275(46):36062-36066.

7. Jiang J, Hui CC. Hedgehog signaling in development and cancer. Dev Cell. 2008;15(6):801-812.

8. Teglund S, Toftgard R. Hedgehog beyond medulloblastoma and basal cell carcinoma. Biochim Biophys Acta. 2010;1805(2):181-208

9. Tremblay MR, McGovern K, Read MA, Castro AC. New developments in the discovery of small molecule Hedgehog antagonists. Curr Opin Chem Biol. 2010;14(3):428-435.

10. Taylor MD, et al. Mutations in SUFU predispose to medulloblastoma. Nat Genet. 2002;31(3):306-310.

11. Zwerner JP, et al. The EWS/FLI1 oncogenic transcription factor deregulates Gli1. Oncogene. 2008;27(23):3282-3291.
12. Rudin CM, et al. Treatment of medulloblastoma with hedgehog pathway inhibitor GDC-0449. N Engl J Med. 2009;361(12):1173-1178.

13. Beauchamp EM, et al. Arsenic trioxide inhibits human cancer cell growth and tumor development in mice by blocking Hedgehog/GLI pathway. J Clin Invest. 2011;121(1):148-160.

14. Beauchamp E, et al. GLI1 is a direct transcriptional target of EWS-FLI1 oncoprotein.J Biol Chem. 2009;284(1):9074.

15. Gore SD, et al. Single cycle of arsenic trioxide-based consolidation chemotherapy spares anthracycline exposure in the primary management of acute promyelocytic leukemia. JClin Oncol. 2010;28(6):1047-1053.

16. Au WY, Tam S, Fong BM, Kwong YL. Determinants of cerebrospinal fluid arsenic concentration in patients with acute promyelocytic leukemia on oral arsenic trioxide therapy. Blood. 2008;112(9):3587-3590.

17. Knipp S, Gattermann N, Schapira M, Kaferstein H, Germing U. Arsenic in the cerebrospinal fluid of a patient receiving arsenic trioxide for relapsed acute promyelocytic leukemia with CNS involvement. Leuk Res. 2007;31(11):1585-1587.

18. Kim J, Lee JJ, Kim J, Gardner D, Beachy PA. Arsenic antagonizes the Hedgehog pathway by preventing ciliary accumulation and reducing stability of the Gli2 transcriptional effector. Proc Natl Acad SciU S A. 2010;107(30):13432-13437.

19. Bai LY, et al. Differential expression of Sonichedgehog and Gli1 in hematological malignancies. Leukemia. 2008;22(1):226-228.

20. Lauth M, Bergstrom A, Shimokawa T, Toftgard R. Inhibition of GLI-mediated transcription and tumor cell growth by small molecule antagonists. Proc Natl Acad Sci U S A. 2007;104(20):8455-8460.

21. Kimura H, Ng JM, Curran T. Transient inhibition of the hedgehog pathway in young mice causes permanent defects in bone structure. Cancer Cell. $2008 ; 13(3): 249-260$

\title{
Gene defects in the soma: some get it and some don't!
}

\author{
Bernice Lo and Michael J. Lenardo
}

Laboratory of Immunology, NIAID, NIH, Bethesda, Maryland, USA.

\begin{abstract}
Advances in DNA sequencing technologies have increased attention on genetic variation in somatic tissues. Although long known to cause neoplastic diseases, somatic variation is now being investigated as a pathogenetic mechanism for other diseases. Somatic changes are genomic DNA variations that were not inherited but arise in tissues throughout life. In this issue of the JCI, Magerus-Chatinet et al. explore somatic changes in patients with autoimmune lymphoproliferative syndrome (ALPS), a congenital disease of defective apoptosis and autoimmunity that is usually associated with germline heterozygous mutations in the gene encoding the Fas death receptor. They explain why certain individuals have severe disease manifestations by documenting somatic alterations in the germline normal FAS allele in an unusual population of "double-negative" $T$ cells found in ALPS. Thus, the oncological concept of somatic loss of heterozygosity leading to selected cell expansion also applies to autoimmune diseases.
\end{abstract}

Conflict of interest: The authors have declared that no conflict of interest exists.

Citation for this article: J Clin Invest. 2011; 121(1):16-19. doi:10.1172/JCI45664.
The pathological role of somatic genetic changes in cancer has long been recognized, but the contribution of somatic mutations in other diseases is only now being uncovered. Somatic mutations are genetic alterations that are not inherited but arise in cells in the body over time. Recent reports have revealed an important role for somatic mutations in a variety of diseases, including ichthyosis with confetti, Wiskott-Aldrich syndrome, and severe combined immunodeficiency syndrome (1-3). Somatic mutations in highly proliferative tissues such as blood and epithelia may be more common than previously appreciated. These somatic events may only be evident when they provide the altered cells with a selective survival or proliferative advantage and cause clinical manifestations in the context of disease. In this issue of the JCI, Magerus-Chatinet and colleagues report on their discovery of how somatic mutations underlie the variable 